\title{
Efficacité d'un traitement intravaginal à base de progestérone chez des vaches croisées Bos indicus x Bos taurus
}

\author{
Justin Kouamo $^{1 *}$ Iliassou ${ }^{1}$ Souley Hayatou ${ }^{2}$ \\ Victor Ngu Ngwa ${ }^{1}$ Camille Teitsa Zangue ${ }^{1}$
}

\begin{abstract}
Mots-clés
Bos indicus, Bos taurus, Goudali, Charolais, produit de croisement, ovulation induite, insémination artificielle, Cameroun
\end{abstract}

Submitted: 27 January 2020

Accepted: 8 September 2020

Published: 25 January 2021

DOI: $10.19182 /$ remvt.31947

\begin{abstract}
Résumé
L'étude a eu pour objectif d'évaluer l'efficacité d'un traitement combiné à base de progestérone intravaginale sur l'induction de l'œstrus de femelles croisées au Cameroun. Un total de 116 vaches Goudali (Bos indicus) x Charolaises (Bos taurus) ayant mis bas au moins une fois, sans antécédent pathologique, avec une note d'état corporel supérieure à 2, ont été sélectionnées dans des élevages du département de la Vina (arrondissement de Nyambaka). Elles ont été rassemblées dans un ranch et réparties en lots de 10 animaux. Le protocole d'induction et de synchronisation des chaleurs a consisté en la pose d'un dispositif intravaginal à base de progestérone (Prid Delta) pendant huit à neuf jours. Vingt-quatre heures avant son retrait, une injection de cinq millilitres de prostaglandine F2alpha (Dinoprost, Enzaprost) a été administrée. Au moment du retrait, 500 UI de gonadotropine chorionique équine ont été injectées. Cinquante-six heures après le retrait, l'insémination a été effectuée à heure fixe avec de la semence de taureaux Gyr et Brahman. Les taux d'induction de l'œstrus et de synchronisation, de rétention du dispositif intravaginal, de gravidité en première insémination, de fécondité, et d'avortement ont été respectivement de $100 \%$, $100 \%$, 56,0 \%, $51,7 \%$, et $4,3 \%$ (en \% des vaches traitées). Le Prid Delta s'est révélé efficace pour l'induction des chaleurs et l'obtention de gravidités chez les vaches Goudali x Charolaises.
\end{abstract}

- Comment citer cet article : Kouamo J., Iliassou, Hayatou S., Ngu Ngwa V., Teitsa Zangue C., 2020. Efficacy of an intravaginal progesterone treatment in crossbred Bos indicus x Bos taurus cows. Rev. Elev. Med. Vet. Pays Trop., 73 (4): 263-268, doi: 10.19182/remvt.31947

\section{INTRODUCTION}

L'élevage bovin au Cameroun occupe une place importante dans le système de production animale avec environ sept millions de têtes. Les bovins représentent $10 \%$ du cheptel global des animaux dans

\footnotetext{
1. Ecole des sciences et de médecine vétérinaire, Université de Ngaoundéré, BP 454, Ngaoundéré, Cameroun.

2. CAMVET-CO SARL, Ngaoundéré, Cameroun.

* Auteur pour la correspondance

Tél. : +237675376954 ; email: justinkouamo@yahoo.fr
}

le pays (Minepia, 2015). Toutefois, la productivité du cheptel reste faible avec des limites d'ordre génétique, zootechnique, sanitaire et de faibles performances de reproduction (Ebangi et al., 2011). Depuis quelques années, les chercheurs de la zone tropicale et subtropicale ont tenté par différents moyens d'augmenter l'efficacité des troupeaux bovins en vue de couvrir les besoins en lait et en viande d'une population dont le taux de croissance est parmi les plus élevés au monde. Les bovins locaux camerounais sont élevés selon un mode traditionnel avec peu de programmes d'amélioration génétique, et en particulier peu de recours à l'insémination artificielle (IA). Cette dernière a été introduite pour la première fois en Afrique en 1935 au Kenya, puis s'est généralisée dans toute l'Afrique subsaharienne à la faveur de différents projets d'amélioration génétique (Kouamo et al., 2009). 
L'IA est peu utilisée au Cameroun alors qu'elle peut être proposée pour augmenter les performances de production bouchère et/ou laitière des races bovines adaptées à la zone tropicale soudano-sahélienne. Malheureusement, la mise en œuvre des programmes d'amélioration génétique par l'IA se heurte à des difficultés diverses notamment la détection des chaleurs et la détermination de la période la plus indiquée pour l'insémination. En effet, les races bovines présentes au Cameroun ont des chaleurs naturelles discrètes et brèves, qui passent souvent inaperçues. Plusieurs protocoles hormonaux conçus pour l'induction, la synchronisation des chaleurs et l'insémination sans détection des chaleurs chez les bovins, à base notamment de progestérone, prostaglandine (PGF2 $\alpha$ ) et gonadotropine chorionique équine (eCG), permettraient de contourner les difficultés liées à la détection de l'œstrus et de regrouper les opérations de surveillance et d'insémination.

L'objectif de ce travail était d'évaluer les effets d'un tel traitement sur des vaches camerounaises dans un programme d'amélioration génétique. Plus spécifiquement, il s'agissait d'évaluer l'induction et la synchronisation des chaleurs ainsi que le taux de réussite de l'insémination par un protocole progestérone-PGF2 $\alpha-\mathrm{eCG}$ chez les femelles croisées Goudali (Bos indicus) x Charolaises (Bos taurus) ; il s'agissait aussi de déterminer les facteurs de variation du taux de réussite de l'IA.

\section{MATERIEL ET METHODES}

\section{Zone d'étude}

L'étude a été réalisée entre décembre 2015 et août 2018 dans la région de l'Adamaoua, département de la Vina, arrondissement de Ngaoundéré $1^{\text {er }}$. L'Adamaoua est située au centre du Cameroun, entre 6 et $8^{\circ}$ $\mathrm{N}$, avec des précipitations moyennes annuelles d'environ 1500 millimètres, et une température moyenne variant de 15 à $29^{\circ} \mathrm{C}$.

\section{Sélection et conduite des vaches}

La présélection des vaches croisées Goudali x Charolaises $(n=160)$ s'est faite dans l'arrondissement de Nyambaka, département de la Vina, puis les femelles retenues $(n=116)$ ont été transférées dans un même ranch de Ngaoundéré $1^{\mathrm{er}}$ et préparées pour le traitement. Les critères de sélection des animaux étaient un examen clinique sans anomalie, une note d'état corporel supérieure à 2 sur une échelle de 1 à 5 (Ferguson et al., 1994) et l'absence d'antécédent pathologique (avortement, dystocie).

Les animaux étaient conduits selon un mode semi-extensif avec huit heures de pâture par jour. Une complémentation était faite uniquement en saison sèche avec une ration à base de tourteaux et de coques de coton, respectivement $1 \mathrm{~kg}$ et $2 \mathrm{~kg}$ par animal et par jour. Le sel était distribué en toute saison. Avant l'arrivée au ranch, les vaches sélectionnées étaient vaccinées contre la pasteurellose (Pastovax), la péripneumonie contagieuse bovine (PPCB) (Perivax), la maladie nodulaire contagieuse (Nodulovax), le charbon symptomatique (Symptovax), et déparasitées à base d'ivermectine (Cevamec $1 \%$ ) et de nitroxynil (Ditrox). A cela, a été ajouté un traitement insecticide et acaricide à base de cyperméthrine (Eradik) deux fois par semaine en saison des pluies et une fois en saison sèche. Aucun taureau ni taurillon n'était présent dans la ferme durant toute l'étude, afin d'empêcher toute reproduction non contrôlée.

\section{Protocole d'induction, de synchronisation de l'aestrus et insémination artificielle}

Toutes les vaches sélectionnées ont été soumises à un diagnostic de non-gravidité par palpation transrectale avant la mise en œuvre du protocole d'induction et de synchronisation des chaleurs. Ce protocole consistait en la pose d'un dispositif intravaginal imbibé de 1,55 g de progestérone Prid Delta (Ceva santé animale, Libourne, France) laissé en place pendant 8-9 jours ; 24 h avant son retrait, une injection intramusculaire (IM) de $5 \mathrm{ml}$ ( $25 \mathrm{mg} / \mathrm{animal})$ de prostaglandine F2alpha (Dinoprost, Enzaprost Ceva santé animale) a été effectuée. Au moment du retrait, une injection de 500 UI d'eCG (Syncro-Part PMSG Ceva santé animale) a été faite par voie IM. Les vaches ont été inséminées de façon systématique (à temps fixe) 56 heures après le retrait du dispositif intravaginal avec de la semence congelée des races $\operatorname{Gyr}(n=46)$ et Brahman $(n=70)$ en provenance du Brésil. Entre le retrait du dispositif et l'IA, les chaleurs ont été observées trois fois par jour (matin, midi et soir) et les signes exprimés par les vaches ont été notés. Durant tout le processus d'IA, les données intrinsèques à l'animal inséminé (âge, poids, note d'état corporel [NEC] à la sélection sur une échelle de 0 à 5 , nombre de lactations et nombre de mois post-partum), et extrinsèques (race du géniteur, taureau géniteur, lieu de dépôt de la semence [ 1 = vagin, $2=$ col de l'utérus, 3 = corps de l'utérus], intervalle pose-retrait du Prid Delta en jours, intervalle retrait Prid Delta-IA en heures et saison) ont été collectées.

\section{Diagnostic de gravidité et suivi après l'insémination artificielle}

Deux mois après l'IA, un diagnostic de gravidité a été effectué par palpation transrectale et les gravidités ont été suivies jusqu'aux mises bas, permettant le calcul de leur durée. Les données relatives aux taux d'induction, de synchronisation, de gravidité, d'avortement, de vêlage et de fécondité ont été obtenues à partir des formules présentées dans le tableau I.

\section{Analyse des données}

Les données collectées ont été saisies dans le tableur Microsoft Excel 2013 et analysées avec le logiciel SPSS 23.0 pour Windows. Le test du Khi carré a permis d'évaluer l'effet des facteurs intrinsèques et extrinsèques à la vache sur le taux de réussite de l'IA. Le test de Kruskal-Wallis a été utilisé pour la comparaison des moyennes. Les différences ont été considérées comme significatives pour des valeurs $\mathrm{p}$ inférieures à $5 \%$.

Tableau I : Formules utilisées pour le calcul de paramètres pour I'induction de l'œestrus et l'insémination artificielle /// Formulas used for calculating parameters for estrus induction and artificial insemination

\begin{tabular}{|c|c|}
\hline Paramètre & Formule \\
\hline \multirow{2}{*}{$\begin{array}{l}\text { Taux } \\
d^{\prime} \text { induction }\end{array}$} & $\mathrm{Nb}$. de femelles venues en chaleurs \\
\hline & $\mathrm{Nb}$. de femelles traitées \\
\hline \multirow[t]{2}{*}{$\begin{array}{l}\text { Taux de } \\
\text { synchronisation }\end{array}$} & $\begin{array}{l}\mathrm{Nb} \text {. femelles traitées ayant } \\
\text { gardé dispositif intravaginal }\end{array}$ \\
\hline & Nb. de femelles traitées \\
\hline \multirow{2}{*}{$\begin{array}{l}\text { Taux de } \\
\text { gravidité }\end{array}$} & $\mathrm{Nb}$. de vaches gravides à 2 mois \\
\hline & Nb. de vaches inséminées \\
\hline \multirow[t]{2}{*}{$\begin{array}{l}\text { Taux } \\
\text { d'avortement }\end{array}$} & $\begin{array}{l}\mathrm{Nb} \text {. de vaches gestantes } \\
\text { ayant perdu leur petit }\end{array}$ \\
\hline & $\overline{\mathrm{Nb} \text {. de vaches inséminées }}$ \\
\hline \multirow[t]{2}{*}{ Taux de vêlage } & $\underline{\mathrm{Nb} \text {. de femelles ayant mis bas }} \times 100$ \\
\hline & Nb. de femelles inséminées \\
\hline \multirow{2}{*}{$\begin{array}{l}\text { Taux de } \\
\text { fécondité }\end{array}$} & $\mathrm{Nb}$. de veaux vivants \\
\hline & $\mathrm{Nb}$. de vaches inséminées \\
\hline
\end{tabular}




\section{RESULTATS}

Au total, 116 inséminations artificielles ont été effectuées pendant la période d'étude. Les moyennes ( \pm écart-type) du poids, de l'âge et du nombre de mois post-partum des femelles sélectionnées étaient respectivement de 331,3 $\pm 51,6 \mathrm{~kg}, 7,4 \pm 2,1$ ans et 7,0 $\pm 0,4$ mois (tableau II).

Sur les 116 synchronisations des chaleurs, aucune vache n'a perdu son système intravaginal entre $\mathrm{J} 0$ (jour de la pose) et $\mathrm{J} 9$ (jour du retrait),

Tableau II : Caractéristiques des vaches croisées Bos indicus $\mathrm{x}$ Bos taurus /// Caracteristics of Bos indicus $x$ Bos taurus crossbred cows

\begin{tabular}{|c|c|c|c|}
\hline & \multicolumn{3}{|c|}{ Données discrètes } \\
\hline & Age (ans) & $\begin{array}{l}\text { Nb. de mois } \\
\text { post-partum }\end{array}$ & Poids (kg) \\
\hline \multirow[t]{3}{*}{$\begin{array}{l}\text { Moy. } \pm \text { ET } \\
\text { (min-max) }\end{array}$} & $\begin{array}{c}7,4 \pm 0,2 \\
(4-5)\end{array}$ & $\begin{array}{c}7,0 \pm 0,4 \\
(2-24)\end{array}$ & $\begin{array}{c}331,3 \pm 4,8 \\
(200-461)\end{array}$ \\
\hline & \multicolumn{3}{|c|}{ Données regroupées en classes } \\
\hline & Modalité & Effectif & $(\%)$ \\
\hline Age (années) & $\begin{array}{c}\leq 5 \\
5-10] \\
>10\end{array}$ & $\begin{array}{c}15 \\
93 \\
8\end{array}$ & $\begin{array}{c}12,9 \\
80,2 \\
6,9\end{array}$ \\
\hline $\begin{array}{l}\text { Note d'état } \\
\text { corporel }\end{array}$ & $\begin{array}{c}3 \\
>3\end{array}$ & $\begin{array}{c}100 \\
16\end{array}$ & $\begin{array}{l}86,2 \\
13,8\end{array}$ \\
\hline $\begin{array}{l}\mathrm{Nb} \text {. de } \\
\text { lactations }\end{array}$ & $\begin{array}{l}{[1-4[} \\
{[4-6]}\end{array}$ & $\begin{array}{l}82 \\
34\end{array}$ & $\begin{array}{l}70,7 \\
29,3\end{array}$ \\
\hline $\begin{array}{l}\text { Nb. de mois } \\
\text { post-partum }\end{array}$ & $\begin{array}{c}{[3-10]} \\
>10\end{array}$ & $\begin{array}{l}97 \\
19\end{array}$ & $\begin{array}{l}83,6 \\
16,4\end{array}$ \\
\hline
\end{tabular}

Moy. \pm ET (min-max) : moyenne \pm écart type (minimum-maximum) /// Moy. \pm ET (min-max): mean \pm standard deviation (minimum-maximum) soit un taux de rétention de $100 \%$. La présence de glaire au niveau de la vulve et une hypertonicité utérine à la palpation transrectale ont été observées chez $100 \%$ des femelles. De même, elles ont toutes chevauché leurs congénères. Les taux d'induction et de synchronisation étaient de $100 \%$.

Deux mois après l'IA, 65 gravidités ont été diagnostiquées, soit un taux de gravidité en première insémination de $56 \%$ (tableau III).

De l'insémination au vêlage, le taux d'avortement clinique (perte du fotus constatée) a été de 4,3\%.

Le nombre de veaux nés vivants a été de 60 à l'issue de l'IA, soit un taux de fécondité de $51,7 \%$

La durée moyenne de gravidité a été de 277,1 \pm 7,6 jours, avec 26,7\% $(\mathrm{n}=16), 60 \%(\mathrm{n}=36)$ et $13,3 \%(\mathrm{n}=8)$ des animaux ayant présenté respectivement une durée inférieure à 270 jours, entre 270 et 285 jours et supérieure à 285 jours.

Les taux de gravidité et de vêlage n'ont pas été influencés par les facteurs intrinsèques étudiés ( $\mathrm{p}>0,05)$, tandis que la durée de gravidité a été significativement longue chez les femelles âgées de 5 à 10 ans et chez celles chez lesquelles le traitement a été mis en place plus de 10 mois après le vêlage $(\mathrm{p}<0,05)$ (tableau III).

Le tableau IV montre que la durée de la gravidité était significativement plus longue chez les vaches dont l'intervalle pose-retrait du Prid Delta était de neuf jours, la semence était déposée dans le col de l'utérus lors de l'IA, et l'insémination avait eu lieu en saison des pluies $(p<0,05)$. Les facteurs extrinsèques n'ont eu aucune influence sur les taux de gravidité et de vêlage $(p>0,05)$.

Sur les 60 naissances, 63,3 \% étaient des mâles (23 Brahman et 15 Gyr) contre $36,7 \%$ de femelles (10 Brahman et 12 Gyr) $(\mathrm{p}=0,29)$. Le poids moyen à la naissance des femelles $(23,5 \pm 0,7 \mathrm{~kg})$ était significativement inférieur à celui des veaux mâles $(24,8 \pm 0,9 \mathrm{~kg})$ $(\mathrm{p}<0,05)$. Les poids moyens à la naissance des veaux issus des vaches inséminées avec des semences de Brahman $(24,5$ $\pm 0,7 \mathrm{~kg})$ et $\mathrm{Gyr}(23,5 \pm 0,8 \mathrm{~kg})$ n'étaient pas significativement différents.

Tableau III : Influence des facteurs intrinsèques sur les taux de gravidité, de vêlage, et sur la durée de gravidité des vaches Bos indicus $\mathrm{x}$ Bos taurus /// Influence of intrinsic factors on pregnancy and calving rates, and pregnancy duration of Bos indicus $x$ Bos taurus cows

\begin{tabular}{|c|c|c|c|c|c|c|c|c|c|c|}
\hline \multirow[t]{2}{*}{ Facteur } & \multirow[t]{2}{*}{ Effectif } & \multicolumn{3}{|c|}{ Gravidité } & \multicolumn{3}{|c|}{ Vêlage } & \multicolumn{3}{|c|}{ Durée de gravidité (jours) } \\
\hline & & $\mathbf{n}$ & $\%$ & $\mathbf{p}$ & $\mathbf{n}$ & $\%$ & $\mathbf{p}$ & $\mathbf{n}$ & Moy. \pm ESM & $\mathbf{p}$ \\
\hline \multicolumn{11}{|c|}{ Classe d'âge (ans) } \\
\hline$\leq 5$ & 15 & 9 & 60,0 & \multirow[t]{3}{*}{0,49} & 9 & 60,0 & \multirow[t]{3}{*}{1,24} & 9 & $268,5^{a} \pm 1,9$ & \multirow[t]{3}{*}{0,002} \\
\hline ]5-10] & 93 & 50 & 53,8 & & 45 & 48,4 & & 45 & $278,8^{b} \pm 1,0$ & \\
\hline$>10$ & 8 & 6 & 75,0 & & 6 & 75,0 & & 6 & $277,3^{\mathrm{ab}} \pm 2,6$ & \\
\hline \multicolumn{11}{|c|}{ Note d'état corporel } \\
\hline 3 & 100 & 56 & 56,0 & \multirow[t]{2}{*}{0,99} & 51 & 51,0 & \multirow[t]{2}{*}{1,00} & 51 & $277,4 \pm 1,1$ & \multirow[t]{2}{*}{0,45} \\
\hline$>3$ & 16 & 9 & 56,2 & & 9 & 56,2 & & 9 & $275,7 \pm 2,6$ & \\
\hline \multicolumn{11}{|c|}{$\mathrm{Nb}$. de lactations } \\
\hline [1-4[ & 82 & 44 & 53,6 & \multirow[t]{2}{*}{0,42} & 41 & 50,0 & \multirow[t]{2}{*}{0,93} & 41 & $276,0 \pm 1,2$ & \multirow[t]{2}{*}{0,11} \\
\hline [4-6] & 34 & 21 & 61,8 & & 19 & 55,9 & & 19 & $279,5 \pm 1,7$ & \\
\hline \multicolumn{11}{|c|}{ Nb. de mois post-partum } \\
\hline [3-10] & 97 & 56 & 57,7 & \multirow[t]{2}{*}{0,41} & 51 & 52,6 & \multirow[t]{2}{*}{0,85} & 51 & $276,1^{\mathrm{a}} \pm 1,1$ & \multirow[t]{2}{*}{0,02} \\
\hline$>10$ & 19 & 9 & 47,4 & & 9 & 47,4 & & 9 & $282,5^{b} \pm 1,3$ & \\
\hline
\end{tabular}

Moy. \pm ESM : moyenne \pm erreur standard de la moyenne; ${ }^{a, b}$ Sur une même colonne, les valeurs suivies de lettres différentes diffèrent significativement (p < 0,05) /// Moy. \pm ESM: mean \pm standard deviation of the mean; ${ }^{a, b}$ On the same column, the values followed by different letters differ significantly $(p<0.05)$ 
Tableau IV : Influence des facteurs extrinsèques sur les taux de gravidité, de vêlage, et sur la durée de gravidité des vaches Bos indicus x Bos taurus /// Influence of extrinsic factors on pregnancy and calving rates, and pregnancy duration of Bos indicus $x$ Bos taurus cows

\begin{tabular}{|c|c|c|c|c|c|c|c|c|c|c|}
\hline \multirow[t]{2}{*}{ Facteur } & \multirow[t]{2}{*}{ Effectif } & \multicolumn{3}{|c|}{ Gravidité } & \multicolumn{3}{|c|}{ Vêlage } & \multicolumn{3}{|c|}{ Durée de gravidité } \\
\hline & & $\mathbf{n}$ & $\%$ & p & $\mathbf{n}$ & $\%$ & $\mathrm{p}$ & $\mathbf{n}$ & Moy. \pm ESM & $\mathbf{p}$ \\
\hline \multicolumn{11}{|l|}{ Race du géniteur } \\
\hline $\begin{array}{l}\text { Brahman } \\
\text { Gyr }\end{array}$ & $\begin{array}{l}70 \\
46\end{array}$ & $\begin{array}{l}36 \\
29\end{array}$ & $\begin{array}{l}51,4 \\
63,0\end{array}$ & 0,22 & $\begin{array}{l}33 \\
27\end{array}$ & $\begin{array}{l}47,1 \\
58,7\end{array}$ & 0,31 & $\begin{array}{l}33 \\
27\end{array}$ & $\begin{array}{l}278,3 \pm 1,4 \\
275,6 \pm 1,3\end{array}$ & 0,13 \\
\hline \multicolumn{11}{|l|}{ Taureau géniteur } \\
\hline 854269COCHISE & 46 & 29 & 60,0 & \multirow[t]{3}{*}{0,41} & 27 & 58,7 & \multirow[t]{3}{*}{0,59} & 27 & $275,6 \pm 1,3$ & \multirow[t]{3}{*}{0,28} \\
\hline CANADIAN SIX X & 58 & 29 & 50,0 & & 26 & 44,8 & & 26 & $278,9 \pm 1,4$ & \\
\hline MADDOX MANSO & 12 & 7 & 58,3 & & 7 & 58,3 & & 7 & $276,1 \pm 4,1$ & \\
\hline \multicolumn{11}{|l|}{ Lieu de dépôt de la semence } \\
\hline 2 (col de l'utérus) & 40 & 21 & 52,5 & \multirow[t]{2}{*}{0,58} & 20 & 50,0 & \multirow[t]{2}{*}{0,49} & 20 & $278,6^{b} \pm 1,2$ & \multirow[t]{2}{*}{0,02} \\
\hline 3 (corps de l'utérus) & 76 & 44 & 57,9 & & 40 & 52,6 & & 40 & $274,1^{\mathrm{a}} \pm 1,4$ & \\
\hline \multicolumn{11}{|c|}{ Intervalle pose-retrait Prid Delta (jours) } \\
\hline $\begin{array}{l}8 \\
9\end{array}$ & $\begin{array}{l}50 \\
66\end{array}$ & $\begin{array}{l}29 \\
26\end{array}$ & $\begin{array}{l}58,0 \\
54,5\end{array}$ & 0,71 & $\begin{array}{l}29 \\
31\end{array}$ & $\begin{array}{l}58,0 \\
46,9\end{array}$ & 0,28 & $\begin{array}{l}29 \\
31\end{array}$ & $\begin{array}{l}274,7^{\mathrm{a}} \pm 1,2 \\
279,4^{\mathrm{b}} \pm 1,4\end{array}$ & 0,005 \\
\hline \multicolumn{11}{|c|}{ Intervalle retrait Prid Delta - IA (heures) } \\
\hline$<56$ & 22 & 15 & 68,2 & \multirow[t]{4}{*}{0,10} & 14 & 63,6 & \multirow[t]{4}{*}{0,03} & 14 & $278,5 \pm 1,4$ & \multirow[t]{4}{*}{0,58} \\
\hline$[56-57[$ & 28 & 14 & 50,0 & & 13 & 46,4 & & 13 & $276,7 \pm 2,0$ & \\
\hline [57-58] & 44 & 20 & 45,4 & & 19 & 43,2 & & 19 & $275,1 \pm 2,3$ & \\
\hline$>58$ & 22 & 16 & 72,7 & & 14 & 63,6 & & 14 & $278,8 \pm 1,8$ & \\
\hline \multicolumn{11}{|l|}{ Saison } \\
\hline Pluvieuse (avril-octobre) & 44 & 24 & 54,5 & \multirow[t]{2}{*}{0,80} & 21 & 47,7 & \multirow[t]{2}{*}{0,32} & 21 & $281,8^{a} \pm 1,1$ & \multirow[t]{2}{*}{0,001} \\
\hline Sèche (novembre-mars) & 72 & 41 & 56,9 & & 39 & 54,2 & & 39 & $274,6^{b} \pm 1,2$ & \\
\hline
\end{tabular}

Moy. \pm ESM : moyenne \pm erreur standard de la moyenne ; IA : insémination artificelle ; ${ }^{a, b}$ Sur une même colonne, les valeurs suivies de lettres différentes diffèrent significativement $(\mathrm{p}<0,05) / / /$ Moy. \pm ESM: mean \pm standard deviation of the mean; IA: artificial insemination; ${ }^{a, b}$ On the same column, the values followed by different letters differ significantly $(p<0.05)$

\section{DISCUSSION}

Les taux d'induction et de synchronisation suite au traitement au Prid Delta ont été comparables à ceux obtenus par Kouamo et al. (2014) chez les femelles zébus Gobra, et les croisées F1 Gobra x Holstein et Gobra x Montbéliard traitées à l'aide du Prid classique. Ils ont été supérieurs à celui obtenu par Okouyi et Hanzen (2016) chez des vaches N'Dama avec le dispositif intravaginal CIDR (37,5\%) contenant 1,38 g de progestérone. O'Hara et al. (2016) montrent que l'administration d'eCG chez les génisses entraînerait une augmentation de la surface du tissu lutéal et des concentrations élevées de progestérone et d'œstradiol sanguin. De plus, la pose d'un dispositif Prid Delta entraînerait une augmentation de la concentration de progestérone chez ces femelles. Ainsi, une injection d'eCG au moment du retrait du Prid Delta permettrait non seulement d'accroitre la production d'œstrogènes à partir du follicule, mais aussi de produire un taux de progestérone sanguin suffisant pour diminuer en début de gravidité le taux de mortalité embryonnaire précoce. Dans notre étude, l'injection de 500 UI d'eCG à la fin du traitement a permis d'induire efficacement l'œstrus chez les vaches Goudali x Charolaises. Leur note d'état corporel (NEC) supérieure ou égale à 3 favorisait une bonne efficacité lors de la mise à la reproduction. En effet, l'efficacité de l'IA dépend de la NEC des vaches au moment de l'IA (Grimard et al., 2003).

Le taux de gravidité de $56 \%$ obtenu chez les femelles Goudali x Charolaises a été comparable à celui rapporté par Bayemi et al. (2014) chez les zébus au Cameroun (52\%), et supérieur à celui de Zongo et al. (2012) chez les zébus Goudali au Burkina Faso (43\%). Ceci pourrait s'expliquer par la participation d'inséminateurs ayant des technicités différentes, un protocole de synchronisation différent (implant et spirale) et par un intervalle retrait spirale-IA qui a varié d'une campagne à l'autre (Kouamo et al., 2014). Wiltbank et al. (2004) montrent qu'une association GnRH, PGF2 $\alpha$, progestérone et œstradiol permettrait le retour des chaleurs des femelles en anœstrus avec un taux de gravidité de $42 \%$.

Le taux d'avortement de $4 \%$ a été inférieur à ceux obtenus par Bayemi et al. (2014), et Foukpê et al. (2018), respectivement chez les femelles zébus multipares au Cameroun et de race Borgou à Okpara au Bénin, soit $20 \%$ et $9 \%$. Cette différence pourrait s'expliquer par l'environnement de l'élevage. Les traumatismes ont été les principales causes d'avortement au cours de notre étude.

Le taux de vêlage $(51,7 \%)$ a été supérieur à celui obtenu par Kouamo et al. (2014) chez les zébus Gobra et croisés F1 au Sénégal (34,6 \%), mais inférieur au taux de 90,5 \% obtenu par Foukpê et al. (2018) au Bénin chez les Borgou. Cette supériorité peut être due à une surestimation du taux de vêlage imputable à la technique de calcul utilisée. En effet, dans ces études, le taux de vêlage est le rapport entre le nombre de vaches ayant vêlé et le nombre de vaches gravides. En revanche, dans notre étude il est défini comme le nombre de vaches ayant vêlé rapporté au nombre de vaches inséminées.

La durée moyenne de gravidité ( $277,1 \pm 7,6$ jours) a été inférieure aux durées de 293,4 $\pm 0,4$ jours et 287,5 $\pm 6,4$ jours obtenues par Messine et al. (2007), et Ledos et Moureaux (2013), respectivement chez la Goudali et la Charolaise. Elle a été significativement plus longue chez les Goudali x Charolaises de plus de cinq ans présentant plus de 10 mois post-partum, dont l'intervalle pose-retrait du Prid Delta a été de neuf jours et chez qui la semence a été déposée dans le col de l'utérus lors de l'IA en saison des pluies $(\mathrm{p}<0,05)$. Ces résultats 
diffèrent de ceux rapportés par Obese et al. (1999) chez la Sanga au Ghana, et Messine et al. (2007) chez la Goudali au Cameroun. Ces auteurs montrent que l'âge de la vache n'a aucune influence sur la durée de la gravidité.

Les inséminations effectuées en saison sèche ont donné de meilleurs résultats (taux de gravidité, taux de vêlage et durée de gravidité) qu'en saison des pluies. En saison sèche, les femelles reçoivent un complément alimentaire, ce qui aurait pour effet d'améliorer leur état et d'augmenter les rendements de reproduction. En saison des pluies, l'humidité relative dans les habitats est élevée, certaines maladies apparaissent telles que les difficultés respiratoires et les affections podales. La pression parasitaire (arthropodes, insectes piqueurs) augmente et l'apparition des facteurs de stress liés à la détérioration de l'environnement physique des animaux pourrait entraîner un dysfonctionnement génital et affecter la fertilité.

Le poids à la naissance est l'expression du développement prénatal de l'animal. Prenant en compte le genre, les veaux étaient plus lourds $(\mathrm{p}<0,05)$ que les velles traduisant un dimorphisme sexuel très prononcé comme rapporté par Koussou et al. (2017). Les poids moyens à la naissance des veaux issus des vaches inséminées avec des semences de Brahman et Gyr étaient inférieurs au poids moyen de 45 kilogrammes des veaux issus des races exotiques, mais supérieurs à ceux des veaux issus des croisements Gobra x Gobra $(20 \mathrm{~kg}$ ) (Kouamo et al., 2014), Arabe x Arabe (22 kg chez le mâle

\section{REFERENCES}

Bayemi P.H., Leinyuy I., Nsongka M.V., Webb E.C., Nchadji J.M., Cavestany D., Perera B.O., 2014. Effect of cow parity and synchronization method with PGF2 $\alpha$ on conception rates of Bos indicus cows in Cameroon. Trop. Anim. Health Prod., 47: 159-162, doi: 10.1007/s11250-014-0701-7

Ebangi A.L., Erasmus G.J., Mbah D.A., Tawah C.L., Ndofor-Foleng H.M., 2011. Evaluation of level of inheritance in the growth traits in the Gudali and Wakwa beef cattle breeds of Adamawa, Cameroon. Livest. Res. Rural Dev., 23 (6): 111-130

Ferguson J.D., Galligan D.T., Thomsen N., 1994. Principle description of body condition score Holstein cow. J. Dairy Sci., 77: 2695-2703, doi: 10.3168/ jds.S0022-0302(94)77212-X

Foukpê Z.A.B., Ibrahim T. A., Soumanou S.T., Ahissou A., Fataou Z.T., Aliyassou M.Y., Bonou G.A., et al., 2018. Reproductive performances of the Borgou cow inseminated on natural or induced estrus with semen from Gir and Girolando at the Okpara Breeding Farm. Vet. World, 11: 693-699, doi: 10.14202/vetworld.2018.693-699

Grimard B., Humblot P., Ponter A.A., Chastant S., Constant F., Mialot J.P., 2003. Efficacité des traitements de synchronisations des chaleurs chez les bovins. Inra Prod. Anim., 16: 211-227, doi : 10.20870/productionsanimales.2003.16.3.3661

Kouamo J., Alloya S., Habumuremyi S., Ouedraogo G.A., Sawadogo G.J., 2014. Evaluation des performances de reproduction des femelles zébus Gobra et des croisés F1 après insémination artificielle en milieu traditionnel dans la région de Thiès au Sénégal. Tropicultura, 32 (2): 8089

Kouamo J., Sow A., Leye A., Sawadogo G.J., Ouedraogo G.A., 2009. Amélioration des performances de production et de reproduction des bovins par l'utilisation de l'insémination artificielle en Afrique Subsaharienne et au Sénégal en particulier : état des lieux et perspectives. RASPA, 7 (3-4): 139148 et $20 \mathrm{~kg}$ chez la femelle) (Koussou et al., 2017). Cette différence serait due à l'effet génétique dont bénéficient les animaux croisés Bos indicus x taurus.

\section{CONCLUSION}

Cette étude a montré qu'un traitement combiné à base de progestérone intravaginale permettrait l'induction de l'œstrus chez les vaches croisées Bos indicus x Bos taurus. Afin d'améliorer leur fertilité, elles doivent être dans un bon état corporel $(\mathrm{NEC}=3)$, complémentées pendant les périodes de soudure (saison sèche). Les performances de reproduction et de production des veaux issus des croisements devront être évaluées en milieu éleveur.

\section{Conflits d'intérêts}

L'étude a été réalisée sans conflit d'intérêts.

\section{Déclaration des contributions des auteurs}

JK et SH ont conçu l'étude; I a recueilli les données et rédigé la première version du manuscrit; JK, VNN, I, CTZ et SH ont participé à la planification de l'étude et révisé le ms.; I et CTZ ont effectué les analyses statistiques ; tous les auteurs autorisent la soumission de la version finale en vue de sa publication.

Koussou M.O., N'Djadody Dingamtar N., Ibrahim K., Kanga Doundom P. 2017. Performances de croissance des veaux zébu arabe à la ferme d'élevage de Mandélia (Tchad). J. Anim. Plant Sci., 33 (1): 5249-5254

Ledos H., Moureaux S., 2013. Durée de gestation chez les principales races de l'espèce bovine, moyenne et variabilité. Compte rendu n 13 78001, Institut de l'élevage, Département Génétique et Phénotypes, Collection résultats, Paris, France, 48 p.

Messine O., Schwalbach L.J.M., Mbah D.A., Ebangi A.L., 2007. Non-genetic Factors Affecting Gestation Length and Postpartum Intervals in Gudali Zebu Cattle of the Adamawa Highlands of Cameroon. Tropicultura, 25 (3): 129-133

Minepia, 2015. Elevage et pêche. In : Annuaire statistique du Cameroun Ministère de I'Elevage, des Pêches et des Industries animales, Yaoundé, Cameroun, 257-268

O’Hara L., Forde N., Duffy P., Randia F., Kelly K., Valenza A., Rodriguez P., et al., 2016. Effect of combined exogenous progesterone with luteotrophic support via equine chorionic gonadotrophin (eCG) on corpus luteum development, circulating progesterone concentrations and embryo development in cattle. Reprod. Fert. Dev., 28 (3): 269-77, doi:10.1071/RD14019

Obese F.Y., Okantah S.A., Oddoye E.O., Gyawu P., 1999. Post-partum reproductive performance of Sanga cattle in small-holder peri-urban dairy herds in the Accra plains of Ghana. Trop. Anim. Health Prod., 31: 181-190

Okouyi M.W.M., Hanzen C., 2016. Effects of insemination timing and GnRH treatment on pregnancy rates of N'Dama cattle after estrus induction with progestin. Rev. Elev. Med. Vet. Pays Trop., 69 (2): 73-78, doi: 10.19182/ remvt.31182

Wiltbank M.C., Lopez H., Gumen A., 2004. Getting Anovular Cows Pregnant. Proceedings Florida Dairy Reprod., 69 - 74

Zongo M., Bayala B., Pitala W., Meyer C., Boly H., Sawadogo L., 2012. Induction d'oestrus et insémination artificielle chez les zébus Azawak et zébus Goudali au Burkina Faso. Tropicultura, 32 (1): 54-61 


\section{Summary}

Kouamo J., Iliassou, Hayatou S., Ngu Ngwa V., Teitsa Zangue C. Efficacy of an intravaginal progesterone treatment in crossbred Bos indicus x Bos taurus cows

The objective of this study was to evaluate the efficacy of a combined treatment based on intravaginal progesterone on the induction of estrus in crossbred cows in Cameroon. A total of 116 Gudali (Bos indicus) x Charolais (Bos taurus) cows having calved at least once, without prior diseases, with a body condition score higher than 2, were selected from farms in Vina Department (Nyambaka District). They were gathered on the same ranch and divided into groups of 10 animals. The heat induction and synchronization protocol consisted of the insertion of a progesterone-based intravaginal device (Prid Delta) for eight to nine days. Twenty-four hours before its removal, an injection of five milliliters of prostaglandin F2alpha (Dinoprost, Enzaprost) was administered. At the time of withdrawal, $500 \mathrm{IU}$ of equine chorionic gonadotropin was injected. Fifty-six hours after withdrawal, insemination was performed at the same fixed time with semen from Gyr and Brahman bulls. The rates of estrus induction and synchronization, intravaginal device retention, pregnancy at first insemination, fertility, and abortion were $100 \%, 100 \%, 56.0 \%, 51.7 \%$, and $4.3 \%$ (as \% of treated cows), respectively. Prid Delta was shown to be effective in inducing heat and obtaining pregnancy in Gudali x Charolais cows.

Keywords: Bos indicus, Bos taurus, Gudali, Charolais, crossbreds, induced ovulation, artificial insemination, Cameroon

\section{Resumen}

Kouamo J., Iliassou, Hayatou S., Ngu Ngwa V., Teitsa Zangue C. Eficiencia de un tratamiento intravaginal a base de progesterona en las vacas cruzadas Bos indicus x Bos taurus

Este estudio tuvo como objetivo el de evaluar la eficiencia de un tratamiento combinado a base de progesterona intravaginal sobre la inducción del estro en hembras cruzadas en Camerún. Un total de 116 vacas Gudali (Bos indicus) x Charolais (Bos taurus) habiendo parido al menos una vez, sin antecedente patológico, con una nota de estado corporal superior a 2, fueron seleccionadas en criaderos del departamento de Vina (distrito de Nyambaka). Fueron reunidas en un rancho y repartidas en lotes de 10 animales. El protocolo de inducción y de sincronización de los calores consistió en la introducción de un dispositivo intravaginal a base de progesterona (Prid Delta) durante ocho a nueve días. Veinticuatro horas antes de retirarlo, se administró una inyección de cinco mililitros de prostaglandina F2alpha (Dinoprost, Enzaprost). Al momento de la extracción, se inyectaron 500 UI de gonadotropina coriónica equina. Cincuenta y seis horas después de la extracción, se efectuó la inseminación artificial a una hora fija con semen de toretes Gyr x Brahman. Las tasas de inducción del estro y de sincronización, de retención del dispositivo intravaginal, de preñez a la primera inseminación, de fecundidad, y de aborto fueron respectivamente de $100 \%, 100 \%, 56,0 \%$, $51,7 \%$, y $4,3 \%$ (en \% de las vacas tratadas). El Prid Delta se reveló eficaz para la inducción de calores y la obtención de preñez en las vacas Gudali x Charolais.

Palabras clave: Bos indicus, Bos taurus, Gudali, Charolais, productos del cruzamiento, ovulación inducida, inseminación artificial, Camerún 